\title{
Management of hyperlipidaemia after coronary revascularisation: follow up study
}

\author{
Etienne Delacrétaz, Petros G Michalopoulos, Juan Ruiz, Hugo Saner, Bernhard Meier
}

We evaluated the management of cardiovascular risk factors focusing on the treatment of hypercholesterolaemia in 245 patients who had had their first coronary revascularisation in a university hospital 1 year earlier.

\section{Subjects, methods, and results}

The patients had had bypass grafting or angioplasty as a first coronary revascularisation procedure. Neither they nor their doctors were informed about the study until 1 year later, when the patients were asked to complete a questionnaire. Serum lipid concentrations were measured, and the modification of cardiovascular risk factors since coronary revascularisation noted.

Data are presented as medians (90\% confidence interval) unless indicated otherwise. Clinical data were analysed either by one way analysis of variance or the Mann-Whitney U test. Categorical variables were compared between groups by a $\chi^{2}$ test. The degree of linear association between variables was evaluated by Pearson's correlation coefficient. Log transformation was used when necessary to normalise distribution. Values of $\mathrm{P}<0.05$ were considered significant.

Overall, $186(76 \%)$ of the 245 patients participated in the study; 98 had had angioplasty and 88 bypass grafting. Of the remainder, 37 refused to participate, 15 were lost to follow up, and 7 had died. Baseline concentrations were total cholesterol $6.1 \mathrm{mmol} / \mathrm{l}(5.9$ to 6.2$)$, high density lipoprotein cholesterol $1.1 \mathrm{mmol} / \mathrm{l}$ (1.1 to 1.2), low density lipoprotein cholesterol $4.1 \mathrm{mmol} / \mathrm{l}(3.9$ to 4.2 ), triglyceride $2.0 \mathrm{mmol} / 1$ (1.9 to 2.1 ), and body mass index $26.6 \mathrm{~kg} / \mathrm{m}^{2}$ (26.1 to 27.1). Serum lipid concentrations were the same 1 year after revascularisation. Ninety four patients had stopped smoking before revascularisation, 29 of the 55 smokers at revascularisation were smoking one year later $(\mathrm{P}<0.005)$; only six of the 26 heavy smokers continued to smoke $>20$ cigarettes daily $(\mathrm{P}<0.001)$. Of all the patients, 180 $(97 \%)$ were receiving antiplatelet or warfarin treatment, or both, whereas only $37(25 \%)$ of the 148 with a serum cholesterol concentration $>5.2 \mathrm{mmol} / 1$ were receiving lipid lowering treatment. After patients over 65 years were excluded, 29 of the $97(30 \%)$ with a serum cholesterol concentration $>5.2 \mathrm{mmol} / \mathrm{l}$ were receiving lipid lowering treatment (figure). Fifty two patients had maintained a low fat diet. Multivariate analysis showed no correlation between adherence to a low fat diet or lipid lowering treatment and either surgical revascularisation-rather than angioplasty-inpatient rehabilitation (89 patients), previous myocardial infarction, low ejection fraction, the presence of symptoms, closer follow up, or satisfaction with treatment.

\section{Comment}

Despite guidelines on the treatment of hypercholesterolaemia in coronary heart disease and recent evidence of the benefit and safety of this treatment, the management of patients after coronary revascularisation in a Swiss

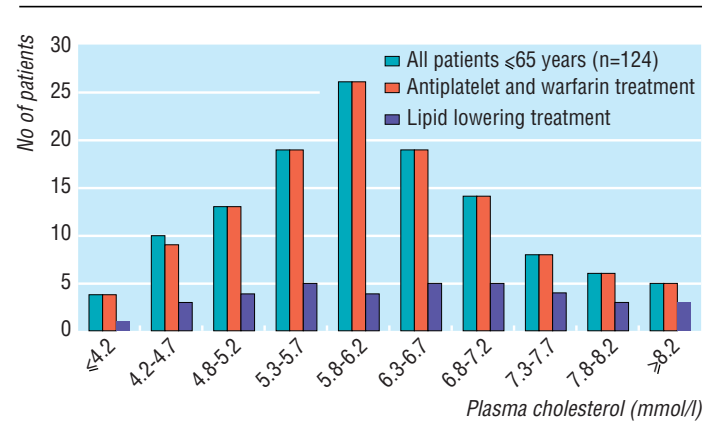

Serum cholesterol concentration 1 year after coronary artery revascularisation in patients $\leqslant 65$ years $(n=124)$, and numbers of patients receiving antiplatelets or warfarin and lipid lowering treatment

university centre was inadequate. ${ }^{1-4}$ Only a few patients with hypercholesterolaemia received adequate lipid lowering treatment and ate a suitable diet 1 year after revascularisation despite strict medical control (during the year, 121 patients visited their doctor 12 or more times and 182 six or more times). In contrast most patients were taking an antiplatelet agent, or warfarin, or both. The difference in the numbers taking lipid lowering treatment or a suitable diet and receiving antiplatelet treatment or warfarin highlights the difference in acceptance of the two prophylactic regimens. This analysis relied on data provided by patients one year after revascularisation. Recommendations by the patients' doctors, rates of drug discontinuation, and the reasons for this ${ }^{5}$ or compliance, were not evaluated.

The causes of this unsatisfactory management need investigation. Doctors performing revascularisation may be concentrating too much on the procedure at the expense of a clear plan for secondary prevention. Both doctors and patients should be made aware of the importance of lipid lowering treatment.

Contributors: ED had the original idea for the study, analysed the data, and wrote the paper; he will act as guarantor for the paper. BM and HS discussed core ideas and helped write the paper. PGM collected the data and helped analyse the data. JR performed the statistical analysis and discussed the presentation of the data.

Funding: None.

Conflict of interest: None.

1 Grundy SM, Bilheimer D, Chait A, Clark LT, Denke M, Havel RJ, et al Summary of the second report of the national cholesterol education program (NCEP) expert panel on detection, evaluation, and treatment of high blood cholesterol in adults (adult treatment panel II). J Am Med Assoc 1993;269:3015-23.

2 Pyorala K, De Backer G, Graham I, Poole Wilson P, Wood D, Breithardt G et al. Prevention of coronary heart disease in clinical practice: recommendations of the task force of the European Society of Cardiology, European Atherosclerosis Society and European Society of Cardiology, European Atherosclerosis Society

3 Brown BG, Zhao XQ, Sacco DE, Albers JJ. Lipid lowering and plaque regression: new insights into prevention of plaque disruption and clinical events in coronary disease. Circulation 1993;87:1781-91.

4 Pedersen TR. Randomised trial of cholesterol lowering in 4444 patients with coronary heart disease: the Scandinavian simvastatin survival study (4S). Lancet 1994:344:1383-9.

5 Luepker R. Current status of cholesterol treatment in the community: the Minnesota heart survey. Am J Med 1997;102:37-42.

(Accepted 28 October 1997)
Cardiology,

University Hospital,

CH-3010 Bern,

Switzerland

Etienne Delacrétaz

cardiology fellow

Petros G

Michalopoulos,

medical student

Hugo Saner,

professor

Bernhard Meier,

professor

Endocrinology

Division, University

Hospital, CH-1011

Lausanne,

Switzerland

Juan Ruiz,

consultant

diabetologist

Correspondence to: Dr E Delacrétaz, Cardiovascular Division, Brigham and Women's

Hospital, Boston,

MA 02115, USA

edelacreta@bics.bwh.

harvard.edu

BMJ 1998;316:1499 